\title{
Osteoradionecrosis of the Mandible and Mastoiditis after Radiotherapy for Parotid Mucoepidermoid Carcinoma
}

\author{
Kumuda Arvind Rao; Shishir Ram Shetty; Subhas G. Babu; and Renita Lorina Castelino
}

\begin{abstract}
Osteoradionecrosis of the mandible in conjunction with mastoiditis is an extremely rare occurrence following irradiation of salivary gland malignancy in the orofacial region. We report one such case of a patient who presented to us with trismus, jaw pain, and ear discharge. Imaging of the jaws revealed classical features of osteoradionecrosis and mastoiditis. This case is important because presenting features like trismus and dental infection led us to investigative procedures that revealed extensive bone involvement including mastoiditis. Trismus progressively increased over a period of 8 years. In this case, we would like to emphasize the importance of good oral hygiene in the postradiotherapy stage for head and neck cancer.
\end{abstract}

Keywords: Mastoiditis; Osteoradionecrosis; Parotid tumors; Radiotherapy

Corresponding Author:

Dr. Kumuda Arvind Rao H.T.

A. B. Shetty Memorial Institute

of Dental Sciences

Nitte University

Deralkatte

Mangalore, Karnataka, India

Phone: 9448696705

Email: kumuda_arht@rediffmail.com

Received: June 13, 2011

Revised: August 3I, 20II

Accepted: August 31, 2011

doi: $10.3121 / \mathrm{cmr} .2011 .1028$ steoradionecrosis (ORN) is a condition of non-vital bone in a site of radiation injury that was first described by Marx $^{1}$ in 1983 as hypovascularity, hypocellularity, and local tissue hypoxia. Although ORN can be spontaneous, it most commonly results from tissue injury. Prior radiation exposure can injure the reserve reparative capacity of bone and cause it to be overwhelmed by apparently innocuous forms of trauma such as denture-related injury, ulcers, or tooth extraction.

While more common in the mandible, ORN is rare in the temporal bone. ${ }^{2}$ The first case of osteoradionecrosis involving the temporal bone was reported in 1952 by $\mathrm{Block}^{3}$ in a patient with syringobulbia. Co-occurrence of ORN in the mandible as well as temporal bone has rarely been reported. It is more common in the mandible than in the maxilla probably because of the richer vascular supply to the maxilla as well as the fact that the mandible is more frequently irradiated. ${ }^{4}$ Herein, we report a rare instance of ORN involving the mandible and mastoid in a patient after radiotherapy for mucoepidermoid carcinoma of the parotid gland.

\section{Case Presentation}

A female patient, aged 38 years, reported to the Department of Oral Medicine and Radiology with the complaint of pain in the right back region of the lower jaw for the past 15 days. The pain was insidious in onset, continuous in nature, and radiated to the right ear. The patient had restricted mouth opening. She also reported having had intermittent discharge from the right ear over the past 5 years. This discharge was initially colorless but later became purulent. The patient had visited an otolaryngologist for the ear discharge on numerous 


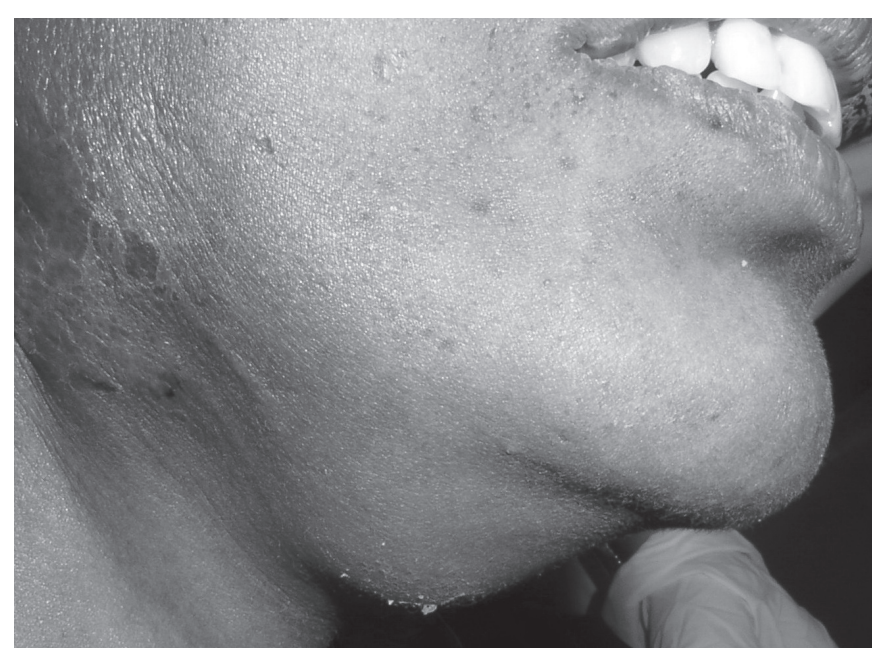

Figure 1. Enlarged right submandibular lymph node.

occasions, and medications had been prescribed, but no reduction in symptoms was observed. The patient had a history of surgery and radiotherapy in the same region for a nodular growth 5 years before presenting with the current complaint. The discharge summary from that time revealed that the treatment modalities were carried out for a low grade mucoepidermoid carcinoma. The patient had reported dryness of the mouth after surgery and radiotherapy, but no history of dizziness, tinnitus, vertigo, or fainting episodes were reported.

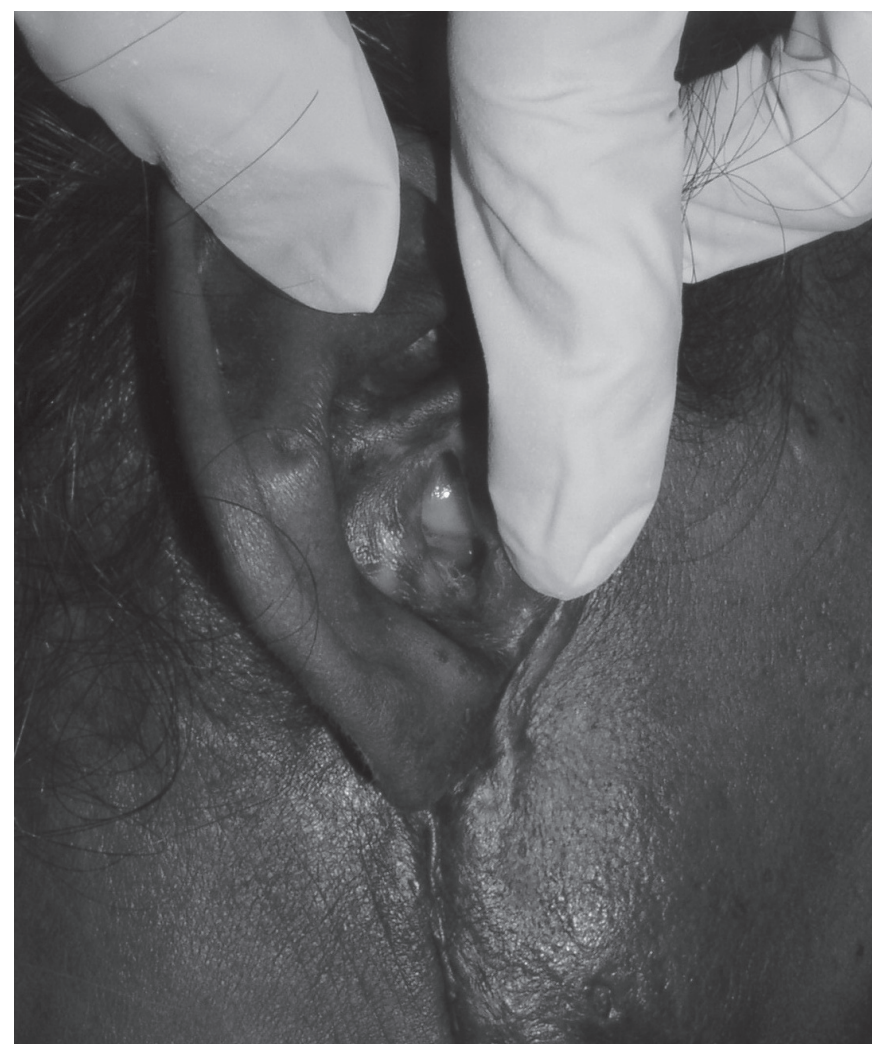

Figure 2. Diffuse purplish black discoloration of the overlying skin and pus discharge from the right external auditory meatus.
On clinical examination bilateral temporomandibular joint movements were restricted with only a $5 \mathrm{~mm}$ mouth opening. Right submandibular lymph node was palpable, enlarged, soft in consistency, tender, and freely movable, measuring about 3 $\mathrm{cm}$ in diameter (figure 1). The extraoral examination revealed diffuse, purplish-black discoloration of the overlying skin in the pre- and post-auricular regions as well as the angle of the mandible of the right side. The skin over the angle of the mandible had a desquamated shiny appearance. A surgical scar extended from the right ear lobe to the base of the mandible. Purulent discharge was noticed in relation to the right external auditory meatus. There were diffuse areas of hyper- and hypo-pigmentation within the ear (figure 2). On palpation, the area was hard in consistency, and the masseter and sternocleidomastoid muscles were fibrosed.

An intraoral examination could not be performed due to restricted mouth opening. An orthopantamogram was performed which revealed multiple decayed teeth in both the upper and lower arches. A mixed radiopaque-radiolucent area was observed distal to tooth 4 and 5 extending to the right condylar area. The upper and lower margins of the right body and the ramus appeared irregular and ill-defined. There was discontinuity in the right lower border of the mandible near the angle region. A retained root stump was also observed in the same region surrounded by irregular, patchy, radiopaque
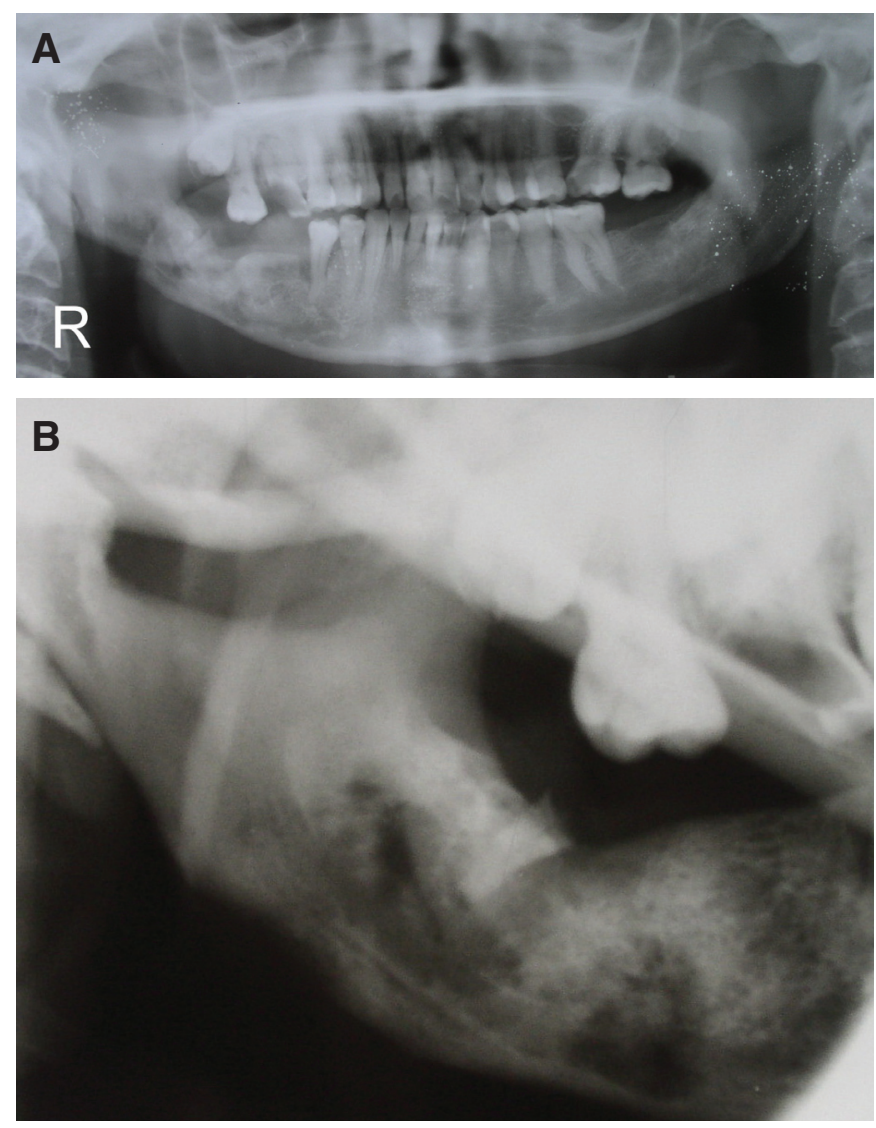

Figure 3. (A) Orthopantamogram showing patchy radiopaque-radiolucent areas in the region of right body and ramus of the mandible. (B) Right lateral oblique view showing extension of lesion into the ramus. 

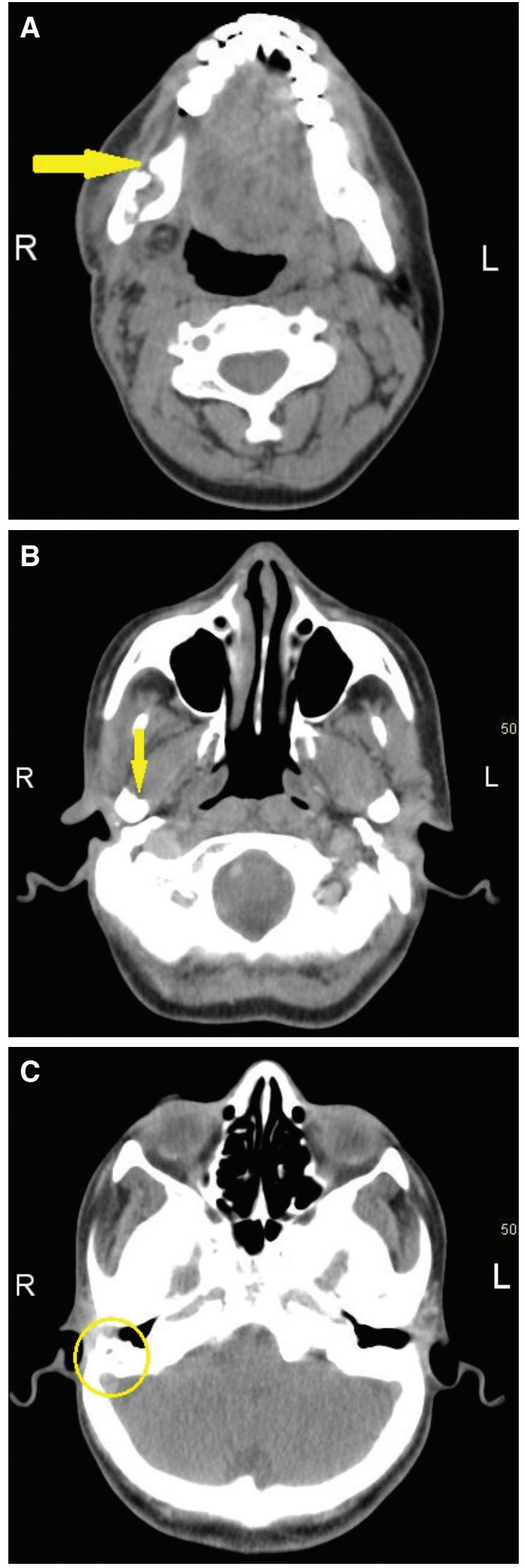

Figure 4. Axial computed tomography section showing (A) right mandibular cortical breach; $(B)$ anterior erosion of the right condyle; and $(\mathrm{C})$ fluid density of right mastoid. and radiolucent areas. The path of the inferior alveolar canal could not be traced through the lesion (figure 3A). Right lateral oblique view of the body of the mandible revealed similar radiographic features (figure 3B). Based on the clinical findings, radiological features, and history of radiotherapy to the head and neck region, a provisional diagnosis of osteoradionecrosis was made. Examination by an otolaryngologist revealed granulation of the tympanic membrane exposing the tympanic plate and necrosis of the overlying skin which was the cause of pus discharge from the ear.

Culture and sensitivity tests were carried out for the pus discharge from the right ear. Following this a course of intravenous penicillin was administered along with antibiotic ear drops. The patient was further referred to a tertiary center for hyperbaric oxygen therapy and is asymptomatic at the present time.

Computed Tomography (CT) with contrast of the head and neck region was performed which showed fluid in the right mandible within the marrow and multiple cortical breaks (figure 4A). There was diffuse infiltrative edema in the right masticator space. The right condylar area also showed marrow edema with anterior erosion of the condyle which enhanced on post-contrast scan (figure 4B). The right mastoid cells showed fluid density consistent with inflammation (figure 4C). There was no evidence of dural breach or extension of infection into the cerebellum or the middle cranial fossa.

Magnetic resonance imaging revealed findings consistent with the CT (figure 5). The right side of the tongue showed fatty atrophy, but the posterior aspect showed high signal intensity on T2 and Short Tau Inversion Recovery images; there was likely extension of the inflammatory process from the mandible. There was hypertrophy of the left half of the tongue, with the asymmetry being secondary to postradiotherapy appearances. The right submental, bilateral submandibular, left level II, III, and level V lymph nodes were visualized. In view of the status of post-radiotherapy for right parotid malignancy, the results were suggestive for osteoradionecrosis with soft tissue infection and right mastoiditis with no evidence of extension of infection into the brain.

Extraction of residual root stump which was the probable source of infection for the ORN was carried out under local anesthesia. The patient was administered empirical antibiotic therapy. Since we did not have the expertise to further manage this complicated case, she was referred to a tertiary center for treatment.

\section{Discussion}

Chronic otomastoiditis may occur as a complication arising from osteoradionecrosis of the temporal bone, as in our case. This may result in hearing loss, gross tissue extrusion, and 
more severe complications including meningitis, facial palsy, intra- or extradural abscesses, pneumocephalus, lateral sinus thrombosis, fistula formation into the parotid gland or temporal mandibular joint, and other cranial neuropathies. ${ }^{5}$ The right temporomandibular joint was involved in our case. In our patient, the disease was relatively diffuse involving mostly the mastoid and the mandible including the condyle and masticator space, but not the cranial base. The patient presented with an exceptionally rare complication of osteoradionecrosis of the temporal bone, secondary to radiotherapy for parotid tumor. On consultation with an otolaryngologist, tympanic membrane perforation was noticed. However, considering the lack of otologic complaints before radiotherapy, the perforation was thought to have been caused by the radionecrosis and/or the subsequent infection.

The incidence of temporal bone ORN is higher after mastoidectomy for facial nerve identification or resection in patients undergoing parotidectomy with postoperative radiotherapy. ${ }^{6}$ The management of osteoradionecrosis in the temporal bone is controversial. In the localized type, conservative treatment with frequent aural cleansing and topical antibiotics is often administered. ${ }^{7}$ Oversew of the ear canal with mastoid obliteration should be considered in this subgroup of patients to avoid this long-term complication of radiotherapy used in the treatment of malignant parotid tumors. ${ }^{6}$

The risk for osteoradionecrosis and infection can be minimized by removing all poorly supported teeth, allowing sufficient time for the extraction wounds to heal before beginning radiation therapy, and adjusting dentures to minimize the risk for denture sores. ${ }^{4}$ Before beginning radiation therapy, all patients should undergo a thorough dental evaluation, including full mouth radiographs, dental and periodontal diagnosis, and prognosis for each tooth. It is generally accepted that meticulous preventive dental treatment should be planned for patients receiving radiotherapy to the head and neck region. ${ }^{8}$ In addition to oral hygiene maintenance with routine dental follow-up, non-surgical or 'conservative' therapy includes nutritional support, topical medicaments, systemic antibiotics, and hyperbaric oxygen. ${ }^{8}$ In 1983, Marx ${ }^{9}$ proposed a staging protocol (subsequently modified) that combined surgery and hyperbaric oxygen for more aggressive treatment of ORN. He was able to achieve complete resolution in all 58 cases. $^{9}$

The management of patients afflicted with ORN of the jaw usually consists of a combination of different modalities and is determined by factors such as the size of the defect, the signs and symptoms of the patient, and the cosmetic and functional derangement consequent to the complication. ${ }^{8}$ For a nonresponsive case, or the diffuse type of osteoradionecrosis, surgical management is indicated. Surgical management of osteoradionecrosis has met with limited success because of the difficulty of accurately assessing the viability of nonnecrotic bone. Failure to resect all nonviable bone results in
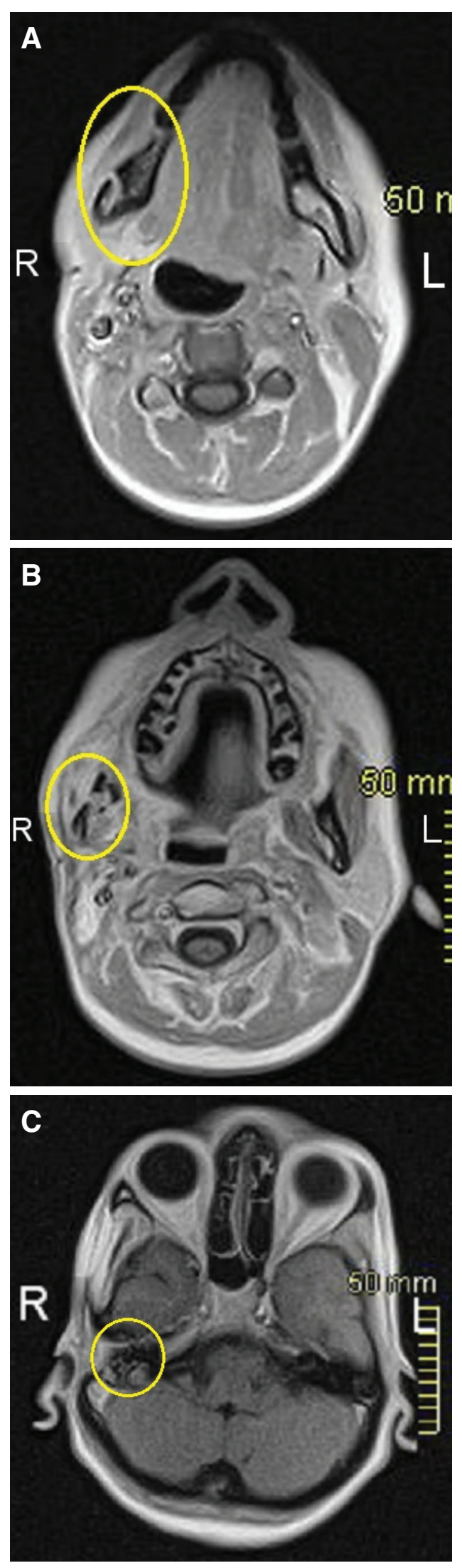

Figure 5. Axial magnetic resonance imaging section showing (A) fluid within marrow with multiple cortical breaks; (B) marrow edema around right condylar head; and (C) fluid density within the right mastoid consistent with inflammation. 
recurrence of a necrotic focus. ${ }^{5}$ Since such expertise was not available in our case, the patient had to be referred to a tertiary center for further management.

Since the introduction of Marx's protocol, ${ }^{9}$ there have been advances in surgical techniques (ie, microvascular surgery), as well as in imaging techniques, that have significantly impacted the diagnosis and management of ORN. High resolution $\mathrm{CT}$ scans and orthopantamograms have become a key component in evaluating and staging ORN before formulating a treatment plan. Reconstructions are now routinely performed immediately after resection of the diseased tissue rather than in a staged fashion. ${ }^{10}$ For advanced ORN of the mandible, radical resection followed by reconstruction using free flap provides a reliable means of obtaining good wound healing with acceptable aesthetic and functional results. ${ }^{11}$

Despite diligent radical treatment, the possibility of recurrent cancer always persists. Extensive osteoradionecrosis with a multiple discharging fistula, a large area of exposed necrotic bone, or a coexistent fracture should be treated primarily with radical sequestrectomy and microvascular free flap reconstruction. Surgery plays a major role in controlling osteoradionecrosis along with adjuvant hyperbaric oxygen therapy. ${ }^{12}$

With the advent of highly precise conformal therapies, such as intensity-modulated radiation therapy, the accurate localization and delivery of radiotherapy is increasingly important. Recent advances in image guided radiotherapy provide increased tumor localization by improving the identification of areas of tumor burden, minimizing the effects of patient setup errors caused by intra-/interfraction motion, and allowing for adaptive replanning due to changes that occur in the tumor or patient during long courses of radiotherapy. These changes are leading to improvements in the therapeutic ratio where doses are increased at diseased sites and minimized at normal tissue sites. ${ }^{13}$

We must also keep in mind that no single imaging modality can provide the entire picture of tumor profiling. Anatomical and functional imaging fusion is essential. Future studies should help determine whether incorporating these and other imaging modalities will improve our ability to cure head and neck cancer and/or reduce the morbidity of the treatment. ${ }^{14}$

\section{Conclusion}

Data currently available regarding osteoradionecrosis are insufficient to assist clinicians faced with its management as well as in counseling patients about the anticipated outcome of therapy. Efforts should be made to monitor patients who have been enrolled in such treatment protocols for long periods of time to evaluate the quality of life and late complications of therapy. This report stresses the importance of educating health care professionals, especially dentists and oral surgeons, of a rare but important side effect of radiation therapy of the head and neck area. It is especially useful in bringing to light the importance of dental evaluation before and after radiation therapy to improve patient treatment outcome.

\section{References}

1. Marx RE. Osteoradionecrosis: a new concept of its pathophysiology. J Oral Maxillofac Surg 1983;41:283-288.

2. Ramsden RT, Bulman CH, Lorigan BP. Osteoradionecrosis of the temporal bone. J Laryngol Otol 1975;89:941-955.

3. Block E. Rontgenschadigung des Schläfenbeins. Z Hals Nas Ohrenheilkd. 1952;3:45-46.

4. White SC, Pharoah MJ. Oral Radiology: Principles and Interpretation. $5^{\text {th }}$ ed. St. Louis, MO: Mosby; 2003.

5. Kveton JF. Surgical management of osteoradionecrosis of the temporal bone. Otolaryngol Head Neck Surg 1988;98: 231-234.

6. Leonetti JP, Marzo SJ, Zender CA, Porter RG, Melian E. Temporal bone osteoradionecrosis after surgery and radiotherapy for malignant parotid tumors. Otol Neurotol 2010;31:656-659.

7. Tsang WS, Ku PK, Andrew van Hasselt C. Osteoradionecrosis of the temporal bone in nasopharyngeal carcinoma after radiotherapy: a case report. Ear Nose Throat J 2000;79: 94-95.

8. Tong AC, Leung AC, Cheng JC, Sham J. Incidence of complicated healing and osteoradionecrosis following tooth extraction in patients receiving radiotherapy for treatment of nasopharyngeal carcinoma. Aust Dent J 1999;44:187-194.

9. Marx RE. A new concept in the treatment of osteoradionecrosis. J Oral Maxillofac Surg 1983;41:351-357.

10. Jacobson AS, Buchbinder D, Hu K, Urken ML. Paradigm shifts in the management of osteoradionecrosis of the mandible. Oral Oncol 2010;46:795-801.

11. Chang DW, Oh HK, Robb GL, Miller MJ. Management of advanced mandibular osteoradionecrosis with free flap reconstruction. Head Neck 2001;23:830-835.

12. Hao SP, Chen HC, Wei FC, Chen CY, Yeh AR, Su JL. Systematic management of osteoradionecrosis in the head and neck. Laryngoscope 1999;109:1324-1327.

13. Nath SK, Simpson DR, Rose BS, Sandhu AP. Recent advances in image-guided radiotherapy for head and neck carcinoma. J Oncol 2009; 2009:752135.

14. Nuyts S. Defining the target for radiotherapy of head and neck cancer. Cancer Imaging 2007;7Spec No A:S50-S55.

\section{Author Affiliations}

Kumuda Arvind Rao HT; ; Shishir Ram Shetty"; Subhas G. Babu*; Renita Lorina Castelino*

*A. B. Shetty Memorial Institute of Dental Sciences, Nitte University, Deralkatte, Mangalore, Karnataka, India 